\title{
The future of pharmaceutics: 3D-printing?
}

\author{
Kinga ILYÉS \\ Department of Pharmaceutical Technology and Biopharmacy, Faculty of Pharmacy, \\ „Iuliu Hatieganu" University of Medicine and Pharmacy, Cluj-Napoca, Romania
}

\begin{abstract}
Over the last century pharmaceutical technology has progressed at an exponential rate, starting off from powder formulation, progressing through tablets, capsules, all the way the nano drug delivery systems. The industrial manufacturing appeared as a result to the increasing demand from the market. Large scale manufacturing setups relied on the one size fits all principle, aiming to serve the populational rather than individual needs. However, there are certain population groups (pediatric, geriatric, poly-medicated etc.) in need of special therapeutic adaptations. Three-dimensional printing (3D-printing, 3DP), due to its versatility and flexibility, can be considered a form of personalized medicine. Dose, release and form, by means of design, can be adapted based on individual needs and preferences, that ultimately can increase therapeutic outcomes, while bringing the manufacturing closer to the patient.
\end{abstract}

Keywords: 3D-printing, personalized medicine, digital health

\section{GENERALITIES}

Three-dimensional printing (3D-printing, 3DP), alternatively called additive manufacturing (AM), as suggested by the nomenclature, involves the layer-bylayer construction of a three-dimensional geometry. The notion of 3DP includes a wide variety of techniques dependent on the main mechanism used in the structure formation. Table 1 gives an overview of the different 3DP techniques, with the most commonly used representative technologies.

In pharmaceutics melt extrusion [1-3], binder jet printing [4-6], SLA $[7,8]$, SLS $[9,10]$ and DLP $[11,12]$ methods are employed. Notably, binder jetting was the first FDA approved 3DP pharmaceutical manufacturing technique via Spirtam ${ }^{\circledR}$ [13]. However, melt extrusion technologies are the most researched in the medical area, with more than $80 \%$ of the articles published in the last five years relying on these technologies [14].
Each technology includes advantages and disadvantages. Products developed by binder jet printing are porous, characterized by rapid disintegration, however the ink viscosity and surface tension has to be controlled within a tight margin as to avoid process interruptions [5]. The use of organic solvents in binder jet printing and SSE is also a risk factor from the pharmaceutical perspective, which may cause stability issues with the active compound along the manufacturing and drying steps [15] and upon ingestion should be limited as per the permitted daily exposure [16]. Although the accuracy of SLA and DLP is superior, there is a limited number of photocrosslinkable polymers available, which are currently not recognized as safe (GRAS) excipients [12]. As for SLS, the limitation factors are the harsh printing conditions (temperature and laser) [10]. FDM is without a doubt the most popular option. The equipment used is small, cheap and easy to handle. The drawbacks are the limitation to use thermal stable 
TABLE 1. The three-dimensional printing techniques [19,20]

\begin{tabular}{|c|c|c|}
\hline 3DP techniques & 3DP construction principle & Representative technologies \\
\hline Material extrusion & $\begin{array}{c}\text { solid or semi-solid based printing, where material is } \\
\text { selectively dispersed through a nozzle }\end{array}$ & $\begin{array}{l}\text { Fused Deposition Modelling (FDM) } \\
\text { Semi-solid extrusion (SSE) }\end{array}$ \\
\hline Direct energy deposition & fusion is done by focused thermal energy (e.g. laser) & Laser Deposition (LD) \\
\hline Powder bed fusion & thermal energy selectively fuses regions of a powder bed & Selective Laser Sintering (SLS) \\
\hline Binder jetting & $\begin{array}{l}\text { inkjet-based printing, where a liquid bonding agent } \\
\text { is selectively deposited to join the powder material } \\
\text { substrate }\end{array}$ & ZipDose ${ }^{\circledR}$ \\
\hline Vat photopolymerization & $\begin{array}{l}\text { liquid photopolymer in a vat is selectively cured by light- } \\
\text { activated polymerization }\end{array}$ & $\begin{array}{c}\text { Stereolithography (SLA) } \\
\text { Digital Light Processing (DLP) }\end{array}$ \\
\hline Material jetting & $\begin{array}{c}\text { droplets of photopolymer build material are selectively } \\
\text { deposited to form layers that are further cured }\end{array}$ & Polyjet Printing (PP) \\
\hline Sheet lamination & sheets of material are bonded & $\begin{array}{l}\text { Laminated Object Manufacturing (LOM) } \\
\text { Ultrasound Additive Manufacturing (UAM) }\end{array}$ \\
\hline
\end{tabular}

materials and necessity to produce filaments, which for pharmaceutical grade excipients is more problematic $[17,18]$.

The manufacturing process is common for all technologies, involving projection and printing. Printing is done based on a previously defined digital design. The Computer Aided Design (CAD) can be created in various $3 \mathrm{D}$ modelling software or by using 3D-scanners, which use a laser to digitally capture the physical characteristics of the object that is desired to be printed. Once projected the design is transferred to the printer, where it is sliced into layers and translated into motion instructions. Additional design characteristics can be added at this level. Hollow structures can be created by infill settings, wall thickness and layer height can be controlled, different segments can be printed with different material substrates, etc.

Although there is an additional need of design, the overall manufacturing flow can be simplified. Complex forms can be produced by a relatively simple technological flow, which in conventional setups may require much more processing steps. Fig. 1 gives an exemplification by using FDM as a reference, which most commonly is used in conjunction with hot melt extrusion (HME).

The easy modulation of the design and the simplicity of the manufacturing process are the key factors that account for the flexibility of the technology.

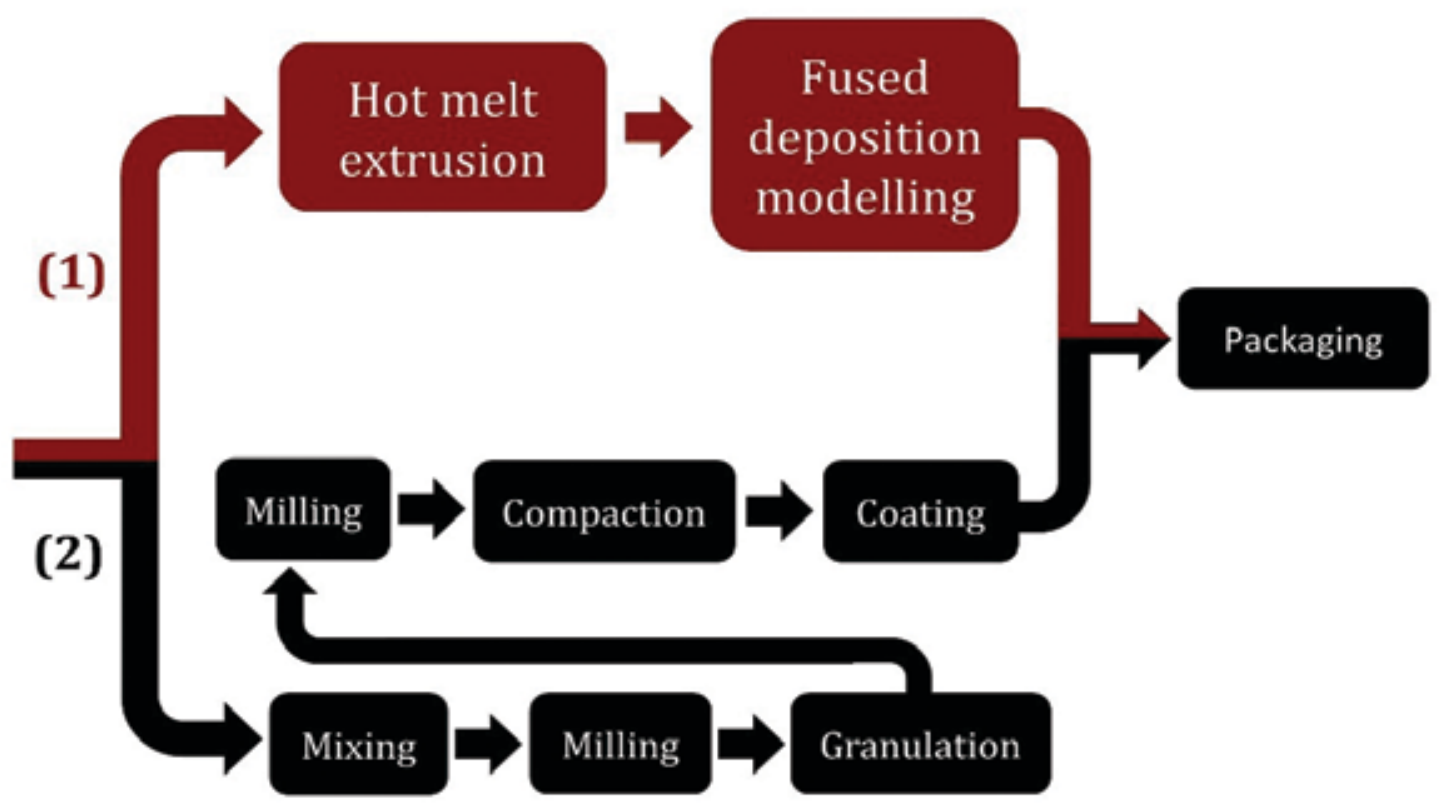

FIGURE 1. The production flow for complex products in modern 3DP (1) and traditional (2) manufacturing 


\section{POTENTIALS}

The aspect which sets apart 3DP from other technologies is the ability to produce complex geometries. This was the characteristics based on which the integration to the medical field was considered. The technology first appeared at the beginning of the 1980s. However, being patent protected, the exploitation was limited for several years. In the medical domain, the first 3DP attempts were related to producing medical devices. The findings along this path are further along, the United States Food and Drug Administration (FDA) having already the Technical Considerations for Additive Manufactured Medical Devices guideline [21] in place to help the integration of the technology.

The integration of 3DP in pharmaceutics is a relatively new research topic. The first publications appeared in $2006[22,23]$, still, it became a hot topic only after the approval of Aprecia's Spirtam ${ }^{\circledR}$ in 2015, which reassured professionals that the pharmaceutical domain was ready to accept 3DP as an alternative manufacturing process. Spirtam ${ }^{\circledR}$ is a Levetiracetam loaded product which is manufactured using the ZipDose $^{\circledR}$, binder jetting technology, and was developed with the purpose to assure rapidly disintegrating high-dose formulations that can be used in imminent epileptic seizures for people that may potentially suffer from dysphagia from the pediatrics to the geriatrics [24].

Pediatrics and geriatrics are special population groups in need of individualized formulations and doses. Due to the flexibility of the 3DP manufacturing process, shape and dose can easily be modulated at design by projecting smaller or bigger structures. Besides material considerations, release can also be controlled by design. By understanding how each design feature contributes to dose and release eventually enables the personalization of the drug delivery systems [25-27].

The variety of shapes that can be obtained by 3DP is high. Conventional and particular geometries can be formed as to for solid oral dosage forms or drug eluting devices. The systems can be adapted to the destined anatomical site or based on preferences. When designing implants, the most essential characteristic is the appropriateness or fit. The systems must fit to the site in order to be tolerable. As such, in some cases, 3D-scanning would be needed in order to obtain a 3D model of an anatomical feature adapted to the morphology of the individual [8]. A large variety of shapes could be produced by different 3DP techniques, e.g. dental fillers with FDM [28], hearing aids with DLP [29], intrauterine devices with SLS [30] etc.

In the same time, shape is a key feature when discussing compliance in children. Starting from this premises, research groups have developed drug delivery systems with Starmix ${ }^{\circledast}$ [31] or cartoon designs [32] and dosage forms that resemble conventional gummies [33]. Based on a previous study, among DLP, FDM, SSE and SLS, DLP was found to produce the most visually appealing printlets for children [34].

For compliance dosing is also important. Having more medications in the therapeutic scheme increases the risk to leave out doses. The most at risk is the polymedicated geriatric population. With 3DP so called polypills can be obtained, which, within the same product, combine several active substances with common or individual release profiles. The most complex product documented was obtained by SLA and combines 6 active substances within the product [35].

\section{PROSPECTS}

The specific notions most used to define the potentials of 3DP are: personalization, print-ondemand, point-of-care. Personalization, as discussed earlier, is due to the potential to adapt design as per individual needs.

Print-on-demand is given by the flexibility of the technology. The equipment used is small, cheap and easy to handle and the technology is simple and flexible, with the possibility of making technology transfer to local manufacturers easy. Most possibly, if approved, 3DP will be used in local hospitals and pharmacies, at point-of-care, bringing the manufacturing process close to the patient. Being able to manufacture at small scale will enable the possibility to personalize pharmaceutical dosage forms based on needs and preferences, but it will also enable the possibility to produce clinical trial samples and orphan drugs [17]. By producing what is necessary in the exact amounts that are required, drug shortages could be prevented, but also unnecessary waste.

3DP can also enable the concept of digital pharmacy [36] potentially completing the digital health cycle 


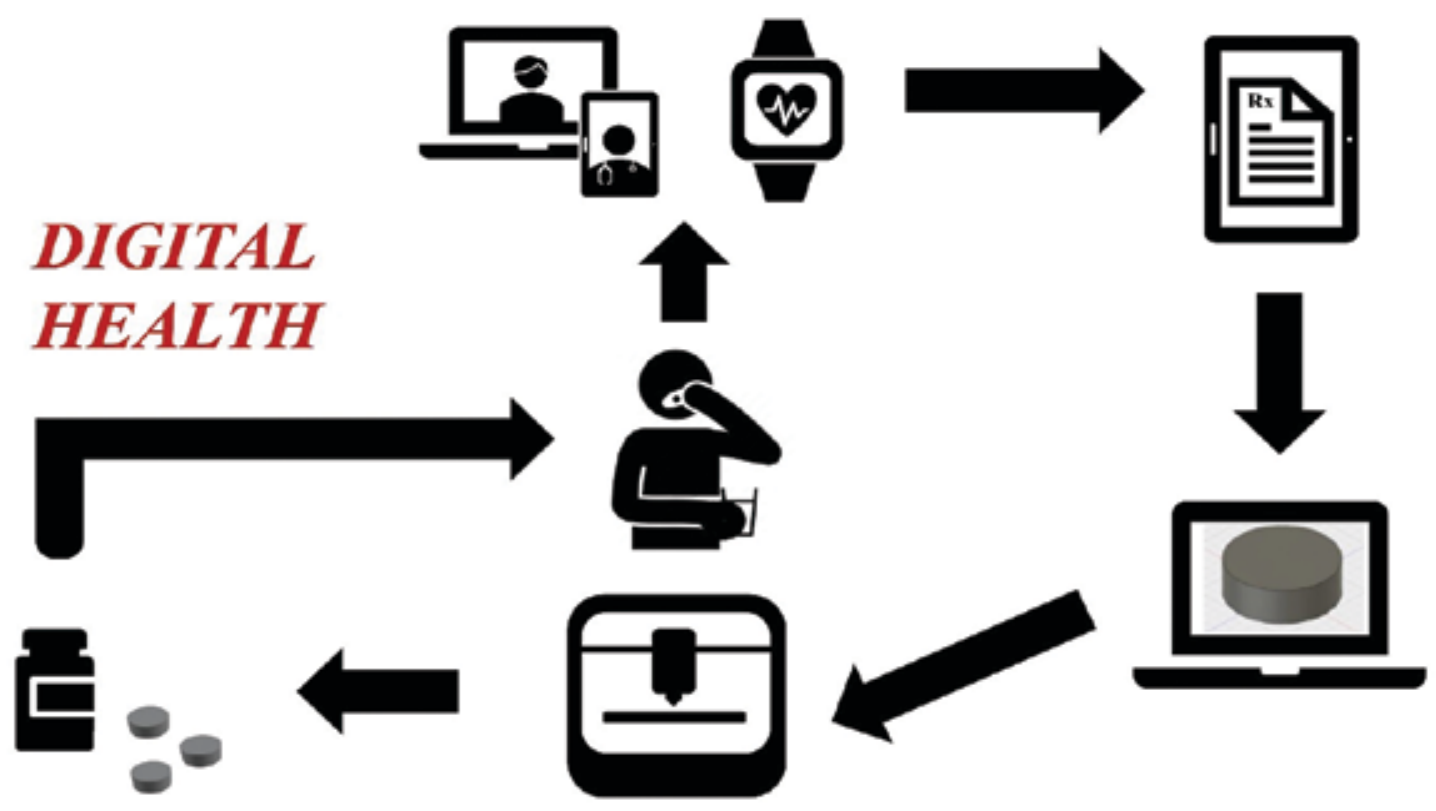

FIGURE 2. The Digital Health concept

(Fig. 2). The other elements included in cycle are telemedicine, with the virtual consultation of the patient, smart devices, that complete the medical decision, and ultimately electronic prescriptions which could guide the manufacturing process.

\section{CONCLUSIONS}

3DP is a technology not to be neglected. The recent proceedings show that in the near future 3DP will infiltrate in several common areas of everyday life.

The outbreak of the coronavirus disease (COVID-19) caused by the SARS-CoV-2 virus highlighted the disadvantage of mass manufacturing and globalization. The supply chain was disrupted, with alarming product shortages appearing in various domains. 3DP came as a rapid response to correct the voids created by the crisis situation. Non-traditional manufacturers and community responders helped to produce personal protective equipment (PPE) and medical devices that were missing from the local market.

In pharmaceutics the technology proposes the shift from populational to individual dosage forms with manufacturing done on small rather than on large scales. During COVID-19 pandemic 3DP might not have been able to help in resolving drug shortages, but the created circumstances might speed up its regulatory acceptance. The therapeutic potentials are diverse and, undoubtably, 3DP is set to revolutionize pharmaceutical manufacturing.

Conflict of interest: none declared Financial support: none declared

\section{REFERENCES}

1. Ilyés, K.; Kovács, N.K.; Balogh, A.; Borbás, E.; Farkas, B.; Casian, T.; Marosi, G.; Tomuță, I.; Nagy, Z.K. The applicability of pharmaceutical polymeric blends for the fused deposition modelling (FDM) 3D technique: Material considerationsprintability-process modulation, with consecutive effects on in vitro release, stability and degradation. Eur. J. Pharm. Sci. 2019, $129,110-123$

2. Li, Q.; Guan, X.; Cui, M.; Zhu, Z.; Chen, K.; Wen, H.; Jia, D.; Hou, J.; $\mathrm{Xu}, \mathrm{W}$.; Yang, X.; et al. Preparation and investigation of novel gastro-floating tablets with 3D extrusion-based printing. Int. J. Pharm. 2018, 535, 325-332.
3. Fanous, M.; Gold, S.; Hirsch, S.; Ogorka, J.; Imanidis, G. Development of immediate release (IR) 3D-printed oral dosage forms with focus on industrial relevance. Eur. J. Pharm. Sci. 2020, 155, 105558.

4. Chang, S.-Y.; Li, S.W.; Kowsari, K.; Shetty, A.; Sorrells, L.; Sen, K.; Nagapudi, K.; Chaudhuri, B.; Ma, A.W.K. Binder-Jet 3D Printing of Indomethacin-laden Pharmaceutical Dosage Forms. J. Pharm. Sci. 2020, 109, 3054-3063.

5. Infanger, S.; Haemmerli, A.; Iliev, S.; Baier, A.; Stoyanov, E.; Quodbach, J. Powder bed 3D-printing of highly loaded drug delivery devices with hydroxypropyl cellulose as solid binder. Int. J. Pharm. 2019, 555, 198-206. 
6. Sen, K.; Manchanda, A.; Mehta, T.; Ma, A.W.K.; Chaudhuri, B. Formulation design for inkjet-based 3D printed tablets. Int. J. Pharm. 2020, 584, 119430.

7. Karakurt, I.; Aydoğdu, A.; Çıkrıkcı, S.; Orozco, J.; Lin, L. Stereolithography (SLA) 3D printing of ascorbic acid loaded hydrogels: A controlled release study. Int. J. Pharm. 2020, 584, 119428.

8. Goyanes, A.; Det-Amornrat, U.; Wang, J.; Basit, A.W.; Gaisford, S. 3D scanning and 3D printing as innovative technologies for fabricating personalized topical drug delivery systems. J. Control. Release 2016, 234, 41-48.

9. Fina, F.; Goyanes, A.; Madla, C.M.; Awad, A.; Trenfield, S.J.; Kuek, J.M.; Patel, P.; Gaisford, S.; Basit, A.W. 3D printing of drug-loaded gyroid lattices using selective laser sintering. Int. J. Pharm. 2018, 547, 44-52.

10. Fina, F.; Goyanes, A.; Gaisford, S.; Basit, A.W. Selective laser sintering (SLS) 3D printing of medicines. Int. J. Pharm. 2017, 529, 285-293.

11. Kadry, H.; Wadnap, S.; Xu, C.; Ahsan, F. Digital light processing (DLP)3D-printing technology and photoreactive polymers in fabrication of modified-release tablets. Eur. J. Pharm. Sci. 2019, 135, 60-67.

12. Madzarevic, M.; Medarevic, D.; Vulovic, A.; Sustersic, T.; Djuris, J.; Filipovic, N.; Ibric, S. Optimization and prediction of ibuprofen release from 3D DLP printlets using artificial neural networks. Pharmaceutics 2019, 11, 544.

13. FDA FDA-Approved Drugs - Spirtam (Aprecia) NDA 207958 Available online: https://www.accessdata.fda.gov/scripts/cder/daf/index. $\mathrm{cfm}$ ?event=overview. process \&AppINo=207958 (accessed on Feb $27,2020)$.

14. Azad; Olawuni; Kimbell; Badruddoza; Hossain; Sultana Polymers for Extrusion-Based 3D Printing of Pharmaceuticals: A Holistic Materials-Process Perspective. Pharmaceutics 2020, 12, 124.

15. Goole J, Amighi K. 3D printing in pharmaceutics: A new tool for designing customized drug delivery systems. Int J Pharm. 2016;499(1-2):376-394.

16. European Medicines Agency ICH guideline Q3C (R6) on impurities: guideline for residual solvents Step 5;

17. Ilyés, K.; Crișan, A.-G.; Porfire, A.; Tomuță, I. Three-dimensional printing by fused deposition modeling (3DP-FDM) in pharmaceutics. Farmacia 2020, 68, 586-596.

18. Shaqour, B.; Samaro, A.; Verleije, B.; Beyers, K.; Vervaet, C.; Cos, P. Production of Drug Delivery Systems Using Fused Filament Fabrication: A Systematic Review. Pharmaceutics 2020, 12, 517.

19. ISO/ASTM 52900 Additive manufacturing - General principles Terminology Available online: https://www.iso.org/obp/ ui/\#iso:std:iso-astm:52900:ed-1:v1:en (accessed on Jul 11, 2020).

20. Trivedi, M.; Jee, J.; Silva, S.; Blomgren, C.; Pontinha, V.M.; Dixon, D.L.; Van Tassel, B.; Bortner, M.J.; Williams, C.; Gilmer, E.; et al. Additive manufacturing of pharmaceuticals for precision medicine applications: A review of the promises and perils in implementation. Addit. Manuf. 2018;23:319-328.

21. FDA CDRH Technical Considerations for Additive Manufactured Medical Devices Guidance for Industry and Food and Drug Administration Staff; 2017;
22. Wang, C.C.; Tejwani, M.R.; Roach, W.J.; Kay, J.L.; Yoo, J.; Surprenant, H.L.; Monkhouse, D.C.; Pryor, T.J. Development of near zero-order release dosage forms using three-dimensional printing (3-DPTM) technology. Drug Dev. Ind. Pharm. 2006, 32, 367-376.

23. Deng, G.Y.; Xiang, L.Y.; Wei, D.H.; Liu, J.; Yun, G.W.; Xu, H. Tablets with material gradients fabricated by three-dimensional printing. J. Pharm. Sci. 2007, 96, 2446-2456.

24. Aprecia | 3D Printing in Medicine Available online: https://www. aprecia.com/ (accessed on Dec 28, 2020).

25. Zhang, J.; Thakkar, R.; Zhang, Y.; Maniruzzaman, M. Structurefunction correlation and personalized 3D printed tablets using a quality by design (QbD) approach. Int. J. Pharm. 2020, 590.

26. Ilyés, K.; Balogh, A.; Casian, T.; Igricz, T.; Borbás, E.; Démuth, B.; Vass, P.; Menyhárt, L.; Kovács, N.K.N.K.; Marosi, G.; et al. 3D floating tablets: Appropriate 3D design from the perspective of different in vitro dissolution testing methodologies. Int. J. Pharm. 2019, 567, 118433.

27. Gorkem Buyukgoz, G.; Soffer, D.; Defendre, J.; Pizzano, G.M.; Davé, R.N. Exploring tablet design options for tailoring drug release and dose via fused deposition modeling (FDM) 3D printing. Int. J. Pharm. 2020, 591, 119987.

28. Yang, Y.; Li, H.; Xu, Y.; Dong, Y.; Shan, W.; Shen, J. Fabrication and evaluation of dental fillers using customized molds via 3D printing technology. Int. J. Pharm. 2019, 562, 66-75.

29. Vivero-Lopez, M.; Xu, X.; Muras, A.; Otero, A.; Concheiro, A.; Gaisford, S.; Basit, A.W.; Alvarez-Lorenzo, C.; Goyanes, A. Antibiofilm multi drug-loaded 3D printed hearing aids. Mater. Sci. Eng. C 2021, 119, 111606

30. Salmoria, G. V.; Vieira, F.E.; Muenz, E.A.; Gindri, I.M.; Marques, M.S.; Kanis, L.A. Additive Manufacturing of PE/fluorouracil/ progesterone intrauterine device for endometrial and ovarian cancer treatments. Polym. Test. 2018, 71, 312-317.

31. Scoutaris, N.; Ross, S.A.; Douroumis, D. 3D Printed "Starmix" Drug Loaded Dosage Forms for Paediatric Applications. Pharm. Res. 2018, 35, 34.

32. Karavasili, C.; Gkaragkounis, A.; Moschakis, T.; Ritzoulis, C.; Fatouros, D.G. Pediatric-friendly chocolate-based dosage forms for the oral administration of both hydrophilic and lipophilic drugs fabricated with extrusion-based 3D printing. Eur. J. Pharm. Sci. 2020, 147.

33. Herrada-Manchón, H.; Rodríguez-González, D.; Alejandro Fernández, M.; Suñé-Pou, M.; Pérez-Lozano, P.; García-Montoya, E.; Aguilar, E. 3D printed gummies: Personalized drug dosage in a safe and appealing way. Int. J. Pharm. 2020, 587.

34. Januskaite, P.; Xu, X.; Ranmal, S.R.; Gaisford, S.; Basit, A.W.; Tuleu, C.; Goyanes, A. I Spy with My Little Eye: A Paediatric Visual Preferences Survey of 3D Printed Tablets. Pharmaceutics 2020, 12, 1100.

35. Robles-Martinez, P.; Xu, X.; Trenfield, S.J.; Awad, A.; Goyanes, A.; Telford, R.; Basit, A.W.; Gaisford, S. 3D printing of a multi-layered polypill containing six drugs using a novel stereolithographic method. Pharmaceutics 2019, 11.

36. Araújo, M.R.P.; Sa-Barreto, L.L.; Gratieri, T.; Gelfuso, G.M.; Cunha-Filho, M.; Araújo, M.R.P.; Sa-Barreto, L.L.; Gratieri, T.; Gelfuso, G.M.; Cunha-Filho, M. The Digital Pharmacies Era: How 3D Printing Technology Using Fused Deposition Modeling Can Become a Reality. Pharmaceutics 2019, 11, 128. 